\title{
Balkanologie
}

Balkanologie Revue d'études pluridisciplinaires

Vol. VII, $n^{\circ} 1$ | 2003

Volume VII Numéro 1

\section{Une zone de libre-échange dans les Balkans a-t-elle un sens?}

\section{Assen Slim}

\section{(2) OpenEdition}

1 Journals

Édition électronique

URL : http://journals.openedition.org/balkanologie/482

DOI : $10.4000 /$ balkanologie.482

ISSN : 1965-0582

Éditeur

Association française d'études sur les Balkans (Afebalk)

Édition imprimée

Date de publication : 1 juin 2003

Pagination : 171-188

ISSN : 1279-7952

\section{Référence électronique}

Assen Slim, « Une zone de libre-échange dans les Balkans a-t-elle un sens? », Balkanologie [En ligne],

Vol. VII, $n^{\circ} 1$ | 2003, mis en ligne le 18 février 2009, consulté le 17 décembre 2020. URL : http:// journals.openedition.org/balkanologie/482 ; DOI : https://doi.org/10.4000/balkanologie.482 


\section{UNE ZONE DE LIBRE-ÉCHANGE DANS LES BALKANS A-T-ELLE UN SENS ?}

Assen SLIM*

En juin 2001, l'intégration régionale dans les Balkans a franchi un nouveau pas : Albanie, Bosnie-Herzégovine, Bulgarie, Croatie, Macédoine, Roumanie et Yougoslavie ont signé un entrelacs d'accords commerciaux bilatéraux. Ces accords s'inspirent des expériences précédentes menées en Europe de l'Est - triangle de Visegrad (décembre 1992), zone de libre-échange de la Baltique (septembre 1993) - et envisagent de bâtir une vaste zone de libreéchange régionale (ZLE) balkanique dans laquelle $90 \%$ des échanges mutuels entre membres seraient exempts de droits de douanes d'ici à 2008.

Les arguments ne manquent pas pour justifier du bien-fondé de cette initiative : rétablir une cohérence commerciale dans la zone des Balkans, mettre en place de nouveaux réseaux de coopération en remplacement de ceux qui ont été détruits par une décennie de conflits, d'embargos et de crises économiques nationales, assurer une meilleure allocation des ressources et contribuer à rationaliser les tissus productifs nationaux.

Toutefois, la constitution d'une zone de libre-échange est toujours un cas particulier isolé dans le temps et dans l'espace. Chaque expérience est unique et soulève des questions théoriques et pratiques spécifiques. La constitution du triangle de Visegrad est intervenue dans un contexte international marqué par la désintégration du CAEM et de l'URSS. Celle de la ZLEB s'est produite à un moment de renaissance de la coopération au sein de la Baltique ${ }^{1}$. Dans chaque cas, le contexte international a été déterminant pour la viabilité de ces zones. Aujourd'hui, le renforcement de l'intégration des Balkans intervient dans un contexte international marqué par l'élargissement de l'UE à l'Europe de l'Est.

\footnotetext{
- Maître de conférences à l'INALCO (assen.slim@inalco.fr).

${ }^{1}$ Bayou (Céline), Chillaud (Matthieu), "L'Europe à la recherche de sa frontière septentrionale", Géoéconomie, (19), Automne 2001.
} 
Cette tendance aura inévitablement des conséquences sur la viabilité de l'intégration des Balkans. Rappelons que Bulgarie et Roumanie, qui devraient adhérer à l'UE à l'horizon 2007, se verront dans l'obligation d'appliquer le tarif extérieur commun (TEC) de l'UE vis-à-vis de leurs actuels partenaires balkaniques ce qui rendra, de fait, caducs les accords signés en juin 2001 avec ces derniers, sauf si l'UE signe à son tour des accords de libre-échange avec les Balkans ${ }^{2}$. Si l'on ajoute à cela le fait que l'UE est déjà le partenaire commercial principal de l'ensemble des pays de la zone de libre-échange des Balkans, on comprend que la viabilité de cette dernière dépendra essentiellement de la nature des accords qui seront conclus entre ces pays et l'UE.

L'intégration économique en cours dans les Balkans est-elle viable ? Quels seront ses effets sur le commerce intra-régional, à court terme, à moyen et long terme?

Dans un premier temps, nous mettrons en lumière l'existence de critères objectifs (théoriques et pratiques) permettant de mesurer la viabilité des zones d'intégration. Dans un second temps, nous utiliserons ces critères opératoires dans le cas de la ZLE des Balkans et proposerons une vision prospective de la région en 2008.

\section{COMMENT MESURER LA VIABILITÉ D'UNE ZONE D'INTÉGRATION ?}

Avec qui et comment participer à une zone d'intégration ? Quels effets et implications économiques en attendre ? Quelles conséquences sur le "bienêtre" régional et mondial sont à prévoir ? Ces questions, soulevées aujourd'hui par l'expérience en cours dans les Balkans, intéressent depuis plus d'un demisiècle les économistes spécialisés sur les relations internationales.

Depuis l'ouvrage fondateur de J. Viner (1950), une littérature considérable s'est développée sur le sujet. Sans revenir ici sur l'ensemble des contributions, nous rappellerons les effets "classiques" (statiques et dynamiques) de la constitution d'une zone de libre-échange (A) et proposerons différents critères opératoires permettant de mesurer la viabilité de ces zones (B).

\section{Les effets "classiques" d'une zone de libre-échange}

J. Viner est le premier à mettre en lumière les effets contraires provoqués par la formation d'une union douanière (zone de libre-échange avec unification du tarif douanier extérieur) entre pays à niveau de développement com-

\footnotetext{
${ }^{2}$ Pour le moment, l'UE a signé un accord commercial d' “association" avec la Macédoine en mars 2001.
} 
parable : les effets de création de commerce (trade-creating effects) et les effets de détournement de commerce (trade-diverting effects) ${ }^{3}$.

Les effets de création de commerce correspondent pour chaque pays membre de l'union au remplacement de productions nationales à coûts unitaires élevés par des importations à coûts unitaires moins élevés en provenance des autres membres de l'union. Notons que la formation de l'union doit également se traduire par une expansion des échanges liée à l'apparition de "flux commerciaux entièrement nouveaux" (wholly new trade selon l'expression de J. Viner ${ }^{4}$ ) qui n'existaient pas avant l'union et qui ne sont pas des substituts à la production nationale. L'ensemble de ces effets (de création et d'expansion des échanges) est censé entraîner une augmentation du "bien-être" régional et mondial. A côté de ces effets "bénéfiques", se manifestent également des effets dits de "détournement" de commerce. Ces derniers renvoient au remplacement d'importations à coûts unitaires faibles en provenance de pays tiers (hors-union) par des importations à coûts unitaires plus élevés mais bénéficiant désormais de tarifs préférentiels (ou d'exemptions de tarif) en provenance de l'union. En conséquence, ils sont censés se traduire par une diminution de bien-être.

En situation d'équilibre partiel ${ }^{5}$, ces effets sont illustrés par le graphique 1 (ci-dessous). Prenons deux pays ( $a$ et $b$ ), tous deux producteurs d'un bien donné (1), ayant décidé de former une zone de libre-échange. Les pays tiers ne participant pas à cette union sont regroupés dans l'ensemble "reste du monde" $(r d m)$. Partons également de l'hypothèse que le pays $a$ appliquait initialement un tarif non discriminatoire $(t)$ vis-à-vis de l'ensemble de ses partenaires commerciaux. Supposons enfin que le prix du bien 1 proposé par $b$ soit plus élevé que le prix des autres partenaires commerciaux de $a$ (soit $P_{b}>P_{r d m}$ ). Dans ces conditions, $\left(P_{b}+P_{b} t\right)$ sera supérieur à $\left(P_{r d m}+P_{r d m} t\right)$ et le pays a se fournira de préférence auprès du $\mathrm{rdm}$. Avant la formation de l'union, le prix du bien 1 pour les consommateurs du pays $a$ est $P_{r d m}(1+t)$ et la quantité importée est $\mathrm{AB}$ au coût $P_{r d m}$ pour la nation.

\footnotetext{
3 Viner (Jacob), The customs union issue, New York: Carnegie Endowment for international peace, 1950, pp. 44-46.

4 Ibid., p. 44.

5 Offre et demande nationales restent inchangées suite à une modification des tarifs douaniers. On parle alors d'effets "statiques"de l'union.
} 


\section{Graphique 1 : Demande d'importations du bien 1 du pays a lors de la formation d'une union douanière avec le pays $b$}

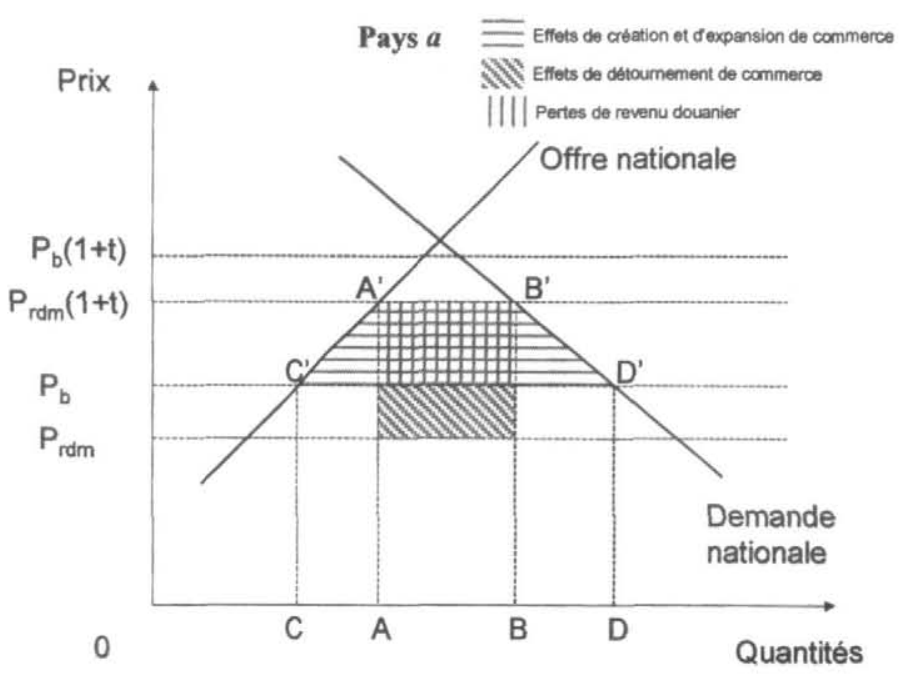

Après la formation de l'union, les droits de douane sont supprimés entre $a$ et $b$ mais ils demeurent à $P_{r d m}(1+t)$ vis-à-vis des pays non-membres de cette dernière. En équilibre partiel, l'offre et la demande nationales ne sont pas affectées par un changement de la politique douanière. La formation de l'union se traduit par une baisse du prix du bien 1 pour les consommateurs de $P_{r d m}$ $(1+t)$ à $P_{b}$, un accroissement de la demande d'importation du pays $a$ en CD et une hausse du coût pour la nation qui passe de $P_{r d m}$ à $P_{b}$ (détérioration des termes de l'échange de $a$ ). Les gains de consommation générés par l'union à travers les effets bruts de création et d'expansion de commerce $\left(\alpha_{a}\right)$ au sens de $J$. Viner correspondent sur le graphique 1 à la surface $A^{\prime} B^{\prime} C^{\prime} D^{\prime}$, soit :

(1) $\alpha_{a \text { (bruts })}={ }_{-}\left(P_{r d m}(1+t)-P_{b}\right)(C A+B D)+\left(P_{r d m}(1+t)-P_{b}\right) A B$

Les pertes provoquées par l'union douanière correspondent aux détournements de commerce $\left(\beta_{a}\right)$ et à la perte des recettes douanières $\left(\mu_{a}\right)$ auparavant prélevées sur les importations en provenance du $r d m$, soit :

(2) $\beta_{a}=\left(P_{b}-P_{r d m}\right) A B$

(3) $\mu_{a}=\left(P_{r d m}(1+t)-P_{b}\right) A B$

La perte de revenu douanier $\left(\mu_{a}\right)$ compense une partie des gains du 
consommateur. En retranchant cette perte de l'équation (1) on obtient les effets nets de création et d'expansion de commerce au sens de J. Viner, soit :
(4) $\alpha_{a \text { (nets })}={ }_{-}\left(P_{r d m}(1+t)-P_{b}\right)(C A+B D)$

L'intérêt ou non de constituer une zone de libre-échange réside donc dans la comparaison entre les effets nets de création et d'expansion du commerce (4) et les effets de détournement (2). Trois cas de figure sont alors possibles : l'union est avantageuse $\left(\alpha_{a \text { (nets })}-\beta a>0\right)$, l'union est désavantageuse ( $\alpha_{a \text { (nets) }}$ $\beta a<0)$, et, enfin, l'union est stérile $\left(\alpha_{a \text { (nets })}-\beta a=0\right)$.

\section{Les critères de viabilité des ZLE}

Les effets nets de création et d'expansion l'emportent-ils systématiquement sur les effets de détournement?

Non, selon J. Viner qui estime qu'aucune réponse d'ordre général ne peut être apportée à cette question. Pour qu'elle ait un sens, la zone de libre-échange doit être avantageuse (cas $n^{\circ} 1$ ), mais, en dernière analyse, seule l'étude concrète de zones intégrées permet de trancher ${ }^{6}$. Toutefois, J. Viner va définir une série de conditions augmentant les chances pour l'union d'être avantageuse. Les principales conditions identifiées de l'auteur sont regroupées ci-dessous et constituent de véritables critères opératoires permettant de prévoir la viabilité des zones d'intégration ${ }^{7}$ :

l'union devra être "la plus large possible " et rassembler des pays à niveau de développement comparable pour une division plus efficace du travail ;

l'union devra adopter un tarif extérieur commun le plus bas possible par rapport au niveau moyen de droits de douanes avant la formation de l'union;

les futurs membres de l'union devront avoir des économies plutôt similaires (fort degré de concurrence) ;

des différences importantes de coûts unitaires de production entre industries des pays membres.

De nombreuses autres conditions sont venues s'ajouter à celles de J. Viner. Dès 1955 , J. E. Meade, qui raisonne comme J. Viner en équilibre partiel, réintroduit dans le raisonnement des effets de production (variation possible des coûts de production, élasticité non infinie de l'offre pour la demande) ${ }^{8}$ à côté

\footnotetext{
6 "Du point de vue du libre-ėchange, les unions douanières ne sont ni nécessairement bonnes ni nécessairement mauvaises", Viner (Jacob), op. cit., p. 52.

7 Ibid., pp. 51-52.

${ }^{8}$ Meade (James E.), The theory of customs union, Amsterdam : North-Holland Publishing Company, 1955 , pp. 35-36, 44-52.
} 
des effets de consommation. Considérant l'analyse de J. Viner incomplète 9 , l'auteur met en lumière des effets complémentaires de détournement des offres d'exportation qui se combinent avec les effets vinériens de détournement des demandes d'importation. Le bien-être se mesure ici à partir de la variation nette du volume du commerce international. L'auteur ajoute ainsi une série de conditions permettant de réduire tant que faire se peut les effets de détournement $^{10}$ :

les futurs membres de l'union devront certes avoir des économies concurrentes, mais présentant un fort degré de "complémentarité potentielle";

le commerce mutuel initial entre membres devra être important avant la formation de l'union;

dans chaque pays membre, offre et demande nationales devront être très réactives l'une par rapport à l'autre (forte élasticité).

Comme J. E. Meade, R. G. Lipsey combine les effets de production et de consommation, mais préfère raisonner en équilibre général ${ }^{11}$. L'auteur considère que les modifications de tarifs provoquées par la formation d'une zone de libre-échange (définie comme l'égalisation des rapports des prix nationaux des biens avec le rapport de leurs prix dans le reste de l'union) $)^{12}$ sont suffisamment importantes pour ne pas être considérées comme marginales au niveau mondial. Dès lors, la clause "toutes choses égales par ailleurs" doit être abandonnée. L'optimum est atteint ici lorsque les taux marginaux de substitution à la consommation entre chaque paire de biens sont égaux aux taux auxquels ces biens peuvent être transformés l'un en l'autre. L'auteur retrouve bien les effets prévus par J. Viner, mais il montre que leur impact sur le "bien-être" est plus complexe qu'on ne l'avait pensé. Les effets de détournement, par exemple, ne se traduisent pas toujours par une réduction du bien-être. Ils peuvent selon les cas accroître ou diminuer la production totale de l'union, celle des pays tiers et/ou celle du monde entier considéré comme un tout et se traduire néanmoins par une amélioration de bien-être. Le graphique 2 illustre ce point.

9 “Comme beaucoup d'idées fondamentales ayant ouvert la voie vers de nouvelles pistes de recherche, l'analyse de Viner est, à mon avis, incomplète à plusieurs égards ; et quand on essaie de la compléter, elle ne reste pas longtemps aussi simple que l'on aurait pu l'imaginer aux premiers abords". Ibid., p. 34.

${ }^{10}$ Ibid., pp. 78, 89-90.

${ }^{11}$ Offre et demande nationales sont affectées par une modification des tarifs douaniers. On parle alors d'effets “dynamiques”de l'union. Lipsey (Richard G.), "The Theory of Customs Union : Trade Diversion and Welfare", Economica, (24), September 1957 ; Lipsey (Richard G.), "The Theory of Customs union : A General Survey", Economic Journal (The), (70), September 1960.

${ }^{12}$ R. G. Lipsey raisonne sur trois biens et trois pays. Le rapport des prix des biens pris deux à deux donne le taux marginal de substitution à la consommation entre chaque paire de bien. (Lipsey (Richard G.), "The Theory of Customs union : A General Survey" (art.cit.), pp. 446-553). 


\section{Graphique 2 : Détournement de commerce et amélioration de bien-être pour le pays $a$}

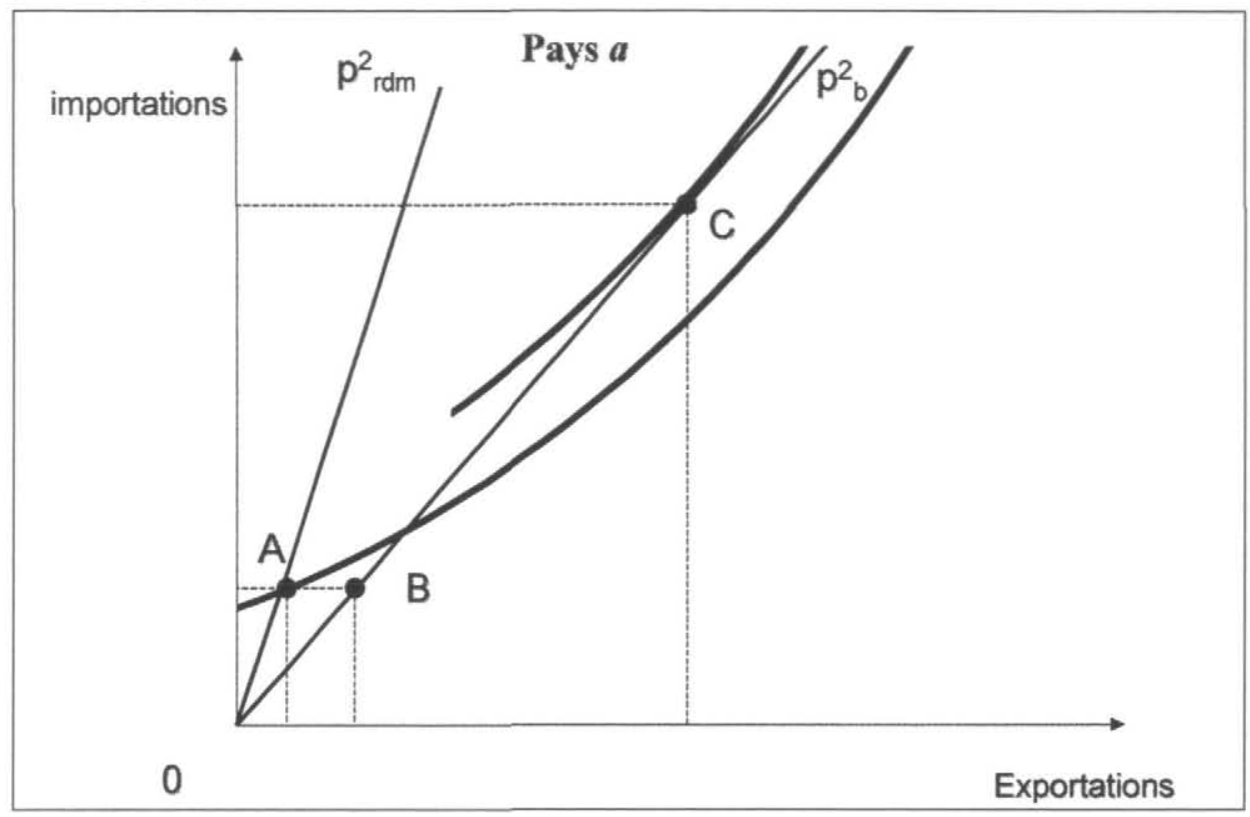

Considérons le même exemple que précédemment en y intégrant un deuxième bien (2). Supposons maintenant que le pays a est spécialisé dans la production et l'exportation du bien 1 et qu'il importe le bien $2 \mathrm{du} r d m$ (au prix $p^{2}{ }_{r d m}$ ) et du pays $b$ (au prix $p^{2}{ }_{b}$ ), l'ensemble des prix étant à présent exprimé en unités de bien 1. Posons enfin comme hypothèse que $p_{r d m}^{2}$ est inférieur à $p^{2}{ }_{b}$. Avant l'union, lorsque le pays $a$ applique un tarif non discriminatoire, alors il achète le bien 2 exclusivement au $\mathrm{rdm}$ au point $\mathrm{A}$ sur le graphique. Après la formation de l'union douanière, si la suppression de tarif entre $a$ et $b$ est suffisante pour compenser le différentiel de prix entre $b$ et $r d m$, alors le pays $a$ se fournira en bien 2 exclusivement auprès de $b$ au point $C$. Dans cet exemple il ne peut y avoir d'effets de création de commerce dans la mesure où $a$ n'est pas spécialisé dans la production du bien 2. En d'autres termes, le passage du point $\mathrm{A}$ au point $\mathrm{C}$ correspond strictement à des effets de détournement de commerce (dans la mesure où le pays $a$ vient de remplacer un fournisseur bon marché par un fournisseur plus coûteux). Toutefois, le passage de $A$ vers $C$ peut être décomposé en deux temps : A vers B puis B vers C. Le point B coupe une courbe d'indifférence inférieure et correspond précisément aux effets de détournement identifiés par J. Viner (les nouveaux flux commerciaux intra-union correspondant strictement aux anciens flux détournés). En revanche, le point $C$ coupe une courbe d'indifférence supérieure à celle du point A en raison de l'ac- 
croissement des importations. Cela revient à dire que, dans certains cas, les effets de détournement de commerce ont un impact positif sur le bien-être. On peut imaginer bien entendu que la courbe d'indifférence passant en A ne croise jamais $p^{2}{ }_{b}$, dans ce cas le passage de $\mathrm{A}$ à $\mathrm{C}$ réduira à tous les coups le bien-être du pays $a$.

R. G. Lipsey conclut qu'il est impossible de dire a priori si la formation d'une zone de libre-échange est avantageuse ou non dans la mesure où l'on ne peut connaître à l'avance l'importance des inégalités induites entre les rapports des prix intra-union et des prix mondiaux $(\mathrm{rdm})$. D. M. Chaffee proposera une généralisation purement algébrique du modèle à trois pays retenu par R. G. Lipsey ${ }^{13}$.

En prolongeant les arguments de R. G. Lipsey, d'autres auteurs sont arrivés à des conclusions proches. H. Bourguinat montre que les processus d'intégration entraînent d'infinies solutions possibles ${ }^{14}$. M. Lutz et P. Wonnacott, en appliquant les arguments de R. G. Lipsey aux seuls biens intermédiaires, montrent que les détournements de commerce n'entraînent pas nécessairement une perte nette d'efficience ${ }^{15}$. En accord avec les conclusions de R. G. Lipsey, C. Kowalczyk montre que certains effets provoqués par la formation de l'union ne sont ni des effets de création, ni des effets de détournement de commerce ${ }^{16}$. Il en va ainsi des achats nouveaux, que l'union réalisera auprès des pays tiers, pour certains produits complémentaires à ceux bénéficiant d'effets de création, d'expansion et de détournement de commerce au sein de l'union (volume-of-trade effects) ${ }^{17}$. C. Kowalczyk insiste également sur les variations (provoquées par la formation de l'union) des prix mondiaux des biens échangés entre les partenaires de l'union et les pays tiers (terms-of-trade effects) ${ }^{18}$. En combinant ces deux effets, $C$. Kowalczyk lève au passage la contradiction apparente entre la proposition de R. G. Lipsey (nécessité d'un commerce mutuel fort avant l'union) et celle de R. Riezman (nécessité d'un commerce mutuel fai-

${ }^{13}$ Chaffee (Donald M. Jr.), "A General Equilibrium Analysis of Trade Creating Customs Unions”, Review of Economic Studies, 42 (2), (130), 1975, p. 279.

14 Bourguinat (Henri), Les marchés communs des pays en développement, Genève : Droz, 1968.

${ }^{15}$ Lutz (Mark), Wonnacott (Paul), "Is there a case for free trade areas", in Schott (Jeff), ed., Free trade areas and U.S. trade policy, Washington : Institute for International Economics, 1989.

${ }^{16}$ Kowalczyk (Carsten), "Welfare and Integration”, International Economic Review, 41 (2), May 2000.

${ }^{17}$ Il est à noter qu'en évoquant "une diffusion générale de la prospérité croissante de l'union douanière", J. Viner suggérait déjà cet effet-volume.

18 "Déterminer empiriquement les effets de détournement de commerce en évaluant les modifications des volumes échangés sans tenir compte des effets prix peut nous conduire à des conclusions erronées sur le bien-être". Kowalczyk (Carsten), art.cit., p. 486 . 
ble avant l'union) puisque désormais tout dépend de la taille des pays formant l'union et de leur capacité à influencer les prix mondiaux ${ }^{19}$.

À partir d'arguments différents de ceux développés par R. G. Lipsey et ses prolongateurs, d'autres auteurs ont complété la théorie des unions douanières. C'est le cas de R. G. Akkihal qui montre que si, suite à la formation de l'union, la nouvelle source d'approvisionnement est plus éloignée du lieu de consommation que ne l'était l'ancienne, alors les effets de création et de détournement de commerce seront moins intenses que ceux prévus par J. Viner ${ }^{20}$. Dans la filiation de R. G. Akkihal, P. Krugman, par exemple, insiste sur la nécessité de tenir compte des économies de coûts de transfert et de communication réalisées lors de la formation de l'union ${ }^{21}$.

Loin des arguments de R. G. Akkihal et de ses prolongateurs, T. Scitovsky considère que quel que soit le sens des effets induits par la formation de l'union, ceux-ci agissent de façon marginale sur le bien-être régional et/ou mondial $^{22}$. Pour T. Scitovsky, l'essentiel de l'union douanière réside dans les mouvements de facteurs de production qu'elle entraîne (l'hypothèse d'immobilité des facteurs est ici levée). L'auteur concentre son attention sur les mouvements de capitaux, à l'origine, selon lui, d'une rationalisation poussée de la production et de la réalisation d'économies d'échelle. L'accroissement des investissements au sein de l'union est censé entraîner une variation des coûts de production (effets de différenciation des coûts de production) et par un accroissement du niveau de vie. Les capitaux étrangers iront de préférence là où le potentiel de retour sur investissement sera le plus prometteur (taille importante du marché, rendement d'échelle significatif, pouvoir d'achat élevé). Comme chez J. Viner, l'élévation du bien-être au sein de l'union devrait exercer à long terme des effets bénéfiques sur le reste du monde.

F. Perroux conteste, quant à lui, l'idée que les effets sur le commerce définis par J. Viner, J. E. Meade et R. G. Lipsey puissent être bénéfiques pour le bienêtre national, régional ou mondial. Pour F. Perroux, l'intégration régionale risque de se traduire non pas par l'élimination des entreprises les moins "effi-

\footnotetext{
19 Ibid., pp. $488-489$.
}

${ }^{20}$ Akkihal (R. G.), "Locational effects in the theory of customs union and welfare analysis" , Indian Economic Journal (The), 21 (1), july-september 1973.

${ }^{21}$ Voir Krugman (Paul R.), "The move to free trade zones" , American Federal Reserve Bank of Kansas City, december 1991 et Krugman (Paul R.), "Regionalism versus multilateralism : analytical notest", World Bank and CEPR Conference, april 1992.

${ }^{22}$ Pour la première année de fonctionnement de la CEE, l'auteur évalue le gain induit par ces effets statiques à 0,005 du PNB des pays de la CEE, contrebalancé par une perte d'un même montant pour le reste du monde. (Voir Scitovsky (Tibor), Economic Theory and Western European Integration, London : George Allen \& Unwin Ltd, 1958, pp. 64-70). 
cientes", mais par les moins "puissantes"23. Cette dernière critique débouche rapidement sur un débat plus général qui est celui de savoir si le libre-échange rapproche du bien-être mondial ou en éloigne...

Quoiqu'il en soit, il ressort de cette revue sommaire de la littérature sur l'intégration : d'une part que plusieurs effets de l'union douanière doivent être pris en compte, tant au niveau national, que régional et mondial ${ }^{24}$ et d'autre part que, sous certaines conditions, les effets de détournement de commerce sont susceptibles d'être réduits ou de conduire, le cas échéant, à une amélioration du bien-être.

Quelle est la portée explicative des développements théoriques précédents lorsqu'on tente de les appliquer à l'intégration au sein des Balkans ?

\section{LA ZLE DES BALKANS EST-ELLE VIABLE ?}

Comme J. Viner et R. G. Lipsey, nous pensons qu'il est impossible de dire, a priori, si la ZLE-Balkans sera avantageuse ou non. Toutefois, la théorie des unions douanières, bien qu'encore sujette à débat, offre une série de critères opératoires qui peuvent être appliqués tels quels pour répondre à cette question. Il faut néanmoins préciser que la situation des Balkans est tout à fait particulière. La décennie 90 a été marquée, comme on le sait, par la désintégration de la RFSY, celle du CAEM, des conflits et guerres, des embargos multiples, des crises nationales sévères. L'ensemble de ces évolutions ne peut être ignoré et "déforme", bien entendu, le fonctionnement normal de la théorie.

\section{Les critères de viabilité de la ZLE des Balkans}

Pour que la ZLE-Balkans ait un sens il faut, comme on l'a vu précédemment, que les effets d'expansion et de création de commerciaux $\left(\alpha_{a(\text { nets }}\right)$ soient maximaux afin de compenser les effets de détournement de commerce $\left(\beta_{a}\right)$. Plusieurs éléments nous laissent à penser que les $\alpha_{a(\text { nets })}$ ne seront pas nuls dans les Balkans. La complémentarité économique est le premier d'entre eux.

\footnotetext{
23 L'une des raisons avancées par l'auteur est l'existence de monopoles, d'oligopoles et de toute une série d'autres "unités actives" qui rendent la concurrence régionale très largement imparfaite. "Quand de très grandes firmes étrangères au moyen d'affiliations et de sous-traitances jouissent d'une zone d'influence, un marché commun leur procure une occasion de réviser en vue de leur meilleure rentabilité leur politique de filiales et de sous-traitances ou leur politique d'alliances avec des alliés mineurs : leur "verdict" n'est à aucun degré celui d'un marchẻ anonyme au service du consommateur" (Perroux (François), Dialogue des monopoles et des nations, équilibre ou dynamique des unités actives, Grenoble : PUG, 1982, p. 80).

24 Pour nous résumer : à côté des effets repérés par J. Viner (effets de création, expansion, détournement de flux commerciaux, variations du surplus des consommateurs et du revenu douanier), il convient d'a-
} 
Rappelons que quatre des sept pays membres de la ZLE-Balkans sont d'anciens membres de la RFSY. Dans ce cadre leur commerce mutuel intérieur (devenu extérieur à partir de 1992) était très intense. Aujourd'hui, ces pays gardent de fortes complémentarités économiques héritées de ce passé commun ${ }^{25}$. La structure sectorielle des échanges intra-ZLE-Blakans (Tableau 1) montre que les quatre pays issus de l'ex-RFSY restent tous spécialisés dans la filière agroalimentaire.

\section{Tableau 1 : Structure par produit des exportations intra-ZLE-Balkans (en \% des exportations intra-ZLE-Balkans, 2000)}

\begin{tabular}{|l|r|r|r|r|r|r|r|}
\hline & $\begin{array}{r}\text { Serbie- } \\
\text { Monténégro }\end{array}$ & $\begin{array}{c}\text { Bosnie- } \\
\text { Herzégovine }\end{array}$ & Croatie & Macédoine & Bulgarie & Roumanie & Albanie \\
\hline Energie & 6,5 & 5,8 & 14,5 & 1,8 & 10,7 & 35,7 & 18,7 \\
\hline Agroalimentaire & 19,5 & 23,1 & 45,0 & 29,2 & 14,6 & 10,3 & 25,3 \\
\hline Textile & 5,4 & 10,7 & 6,6 & 9,7 & 5,2 & 2,6 & 1,1 \\
\hline Bois, papier & 3,3 & 9,5 & 5,2 & 2,9 & 5,8 & 3,5 & 6,6 \\
\hline Produits chimiques & 16,1 & 6,9 & 17,3 & 21,8 & 29,2 & 18,4 & 8,8 \\
\hline Sidérurgie & 1,8 & 3,2 & 0,8 & 11,3 & 11,2 & 11,8 & 2,2 \\
\hline Métaux non-ferreux & 13,6 & 12,3 & 0,6 & 7,6 & 9,7 & 6,6 & 26,4 \\
\hline Mécanique & 7,6 & 3,8 & 4,4 & 6,7 & 6,7 & 3,9 & 4,4 \\
\hline Véhicules & 7,4 & 3,9 & 0,6 & 2,4 & 0,8 & 5,9 & 1,1 \\
\hline Matériel électrique & 2,8 & 1,7 & 3,5 & 5,7 & 2,8 & 1,0 & 1,1 \\
\hline Electronique & 0,5 & 0,2 & 1,5 & 0,5 & 2,7 & 0,1 & 1,1 \\
\hline Autres & 15,5 & 18,9 & 0,0 & 0,4 & 0,6 & 0,2 & 3,2 \\
\hline TOTAL & 100,0 & 100,0 & 100,0 & 100,0 & 100,0 & 100,0 & 100,0 \\
\hline
\end{tabular}

Source : calculé à partir des données de CHELEM

Ce profil de spécialisation doit être interprété comme une source de complémentarité économique (J. E. Meade) et non de concurrence (J. Viner) dans la mesure où ces quatre pays restent leurs principaux fournisseurs et clients dans la filière agroalimentaire : la Macédoine absorbe $68,8 \%$ des exportations de produits agroalimentaires de la Serbie-Monténégro qui elle-même absorbe $61,5 \%$ de celles de la Macédoine et $64,5 \%$ de celles de la Bosnie-Herzégovine.

jouter des effets "termes de l'échange" additionnels (variations des prix entre l'union et le reste du monde), des effets "volume"( changement dans les volumes échangés entre l'union et le reste du monde), des effets de "différenciation des coûts" (liés aux mouvements de capitaux au sein de l'union).

${ }_{25}$ Pour la cas de la Macédoine, voir par exemple Miljovski (Jane), Uzunov (Vanco), International and Regional Economic Integration in South East Europe : The Case of Macedonia, Document de travail disponible à l'adresse suivante : http://www.wiiw.ac.at/balkan/regionalism.html,2001. 
Cette dernière achète à son tour 90,5\% des exportations agroalimentaires de la Croatie ${ }^{26}$.

Cette complémentarité est renforcée par les spécialisations différentes des trois autres pays de la ZLE-Balkans : la Bulgarie est spécialisée sur les produits chimiques $(43,8 \%$ de ses exportations de produits chimiques allant vers la Macédoine), la Roumanie sur l'énergie ( 68,2 de ses exportations d'énergie allant vers la Bulgarie) et l'Albanie sur les métaux non-ferreux (66,7 \% de ses exportations de métaux non-ferreux allant vers la Macédoine).

Selon J. Viner, la complémentarité intra-ZLE-Balkans aurait toutes les chances de se traduire par des effets bénéfiques de création et d'expansion des échanges. D'autant que les niveaux de développement des pays de la ZLEBalkans sont semblables. Des pays proches du point de vue de leur développement économique présentent des tissus productifs "compatibles les uns avec les autres et sont donc susceptibles d'instaurer entre eux des investissements croisés importants et des échanges mutuels intenses ${ }^{27}$ ".

Un simple indicateur de PIB/hab. confirme la proximité en matière de développement de ces pays. La figure 1 montre toutefois que deux pays se distinguent significativement des autres : il s'agit de la Bulgarie et de la Croatie. Leur niveau plus élevé de PIB/hab. reflète les conditions relativement plus avantageuses auxquelles seront confrontés les exportateurs et les investisseurs étrangers.

\section{Figure 1 : PIB/hab. en USD constants en 2001 (USD de 1995)}

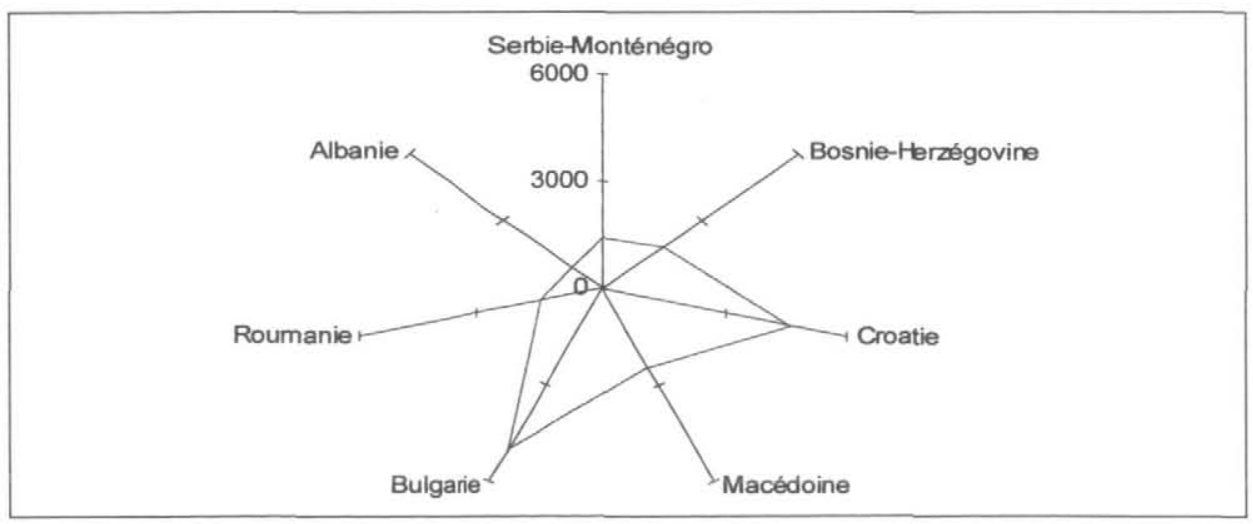

Source : calculé à partir des données de CHELEM

${ }^{26}$ Calculs réalisés pour l'année 2000 à partir de la base de données CHELEM. Si les pays avaient été concurrents, ils n'auraient pas été leurs principaux clients et fournisseurs au sein d'une seule et même filière (agroalimentaire).

27 Viner (Jacob), op. cit. 
L'utilisation d'indicateurs plus représentatifs du "développement" confirme cette inégalité. Le classement réalisé par le PNUD sur la base de l'indicateur du développement humain (IDH) ne dément pas la primauté de la Bulgarie et de la Croatie dans la région. L'IDH mesure le niveau atteint par un pays en termes d'espérance de vie, d'instruction et de PIB/hab. Le classement du FMI, qui tient compte du PIB, de la balance des transactions courantes et des réserves de change place la Roumanie en tête ${ }^{28}$.

\section{Tableau 2 : Classements des Balkans par le PNUD et par le FMI en 2000}

\begin{tabular}{|l|c|c|}
\hline Pays & Rang IDH du PNUD & "Quotas" du FMI (en DTS) \\
\hline Croatie & 49 & 823,0 \\
\hline Bulgarie & 60 & 640,2 \\
\hline Roumanie & 64 & 1030,2 \\
\hline Macédoine & 69 & 68,9 \\
\hline Albanie & 94 & 48,7 \\
\hline Serbie-Monténégxo & - & - \\
\hline Bosnie-Herzégovine & - & - \\
\hline
\end{tabular}

Source : PNUD, Rapport mondial sur le développement humain (2000) et www.imf.org

En d'autres termes, les effets de création et d'expansion des échanges seront très inégalement répartis, bénéficiant en priorité aux pays les mieux classés en matière de développement.

Enfin, on peut ajouter que la proximité géographique des pays membres de la ZLE-Balkans est susceptible de réduire les coûts de transaction (logistique, transport, communication, etc.) et de jouer en faveur des effets de création et d'expansion du commerce ${ }^{29}$.

\section{LES LIMITES DE LA ZLE DES BALKANS}

Toutefois, un grand nombre de facteurs nous conduisent à la plus grande prudence sur l'avenir de la ZLE-Balkans.

En premier lieu, une répartition équilibrée des bienfaits de l'intégration implique que les pays membres aient des tailles économiques comparables

\footnotetext{
${ }^{28}$ Pour un commentaire de l'indicateur du FMI, lire Minassian (Garabed), "The Economic Environnement in Albania, Bulgaria, Macedonia FYR, and Greece : A Cross-Country Study", Eastern European Economics, 40 (4), july-august 2002, pp. 46-47,79.
}

29 Akdcihal (R. G.), art.cit.; Krugman (Paul R.), "The move to free trade zones " (art.cit). 
afin d'absorber de la même manière le "choc" de l'intégration ${ }^{30}$. En cas de différence de taille trop marquée, le risque est de voir les petits pays enregistrer un maximum d'effets de détournement alors que les gros bénéficieraient plutôt des effets de création et d'expansion des échanges. Parmi les différents indicateurs de taille couramment utilisés, nous avons choisi de classer les pays en fonction de leur population et de leur PIB en valeur absolue.

\section{Figure 2 : C lassement des pays selon leur PIB et leur population en 2001}

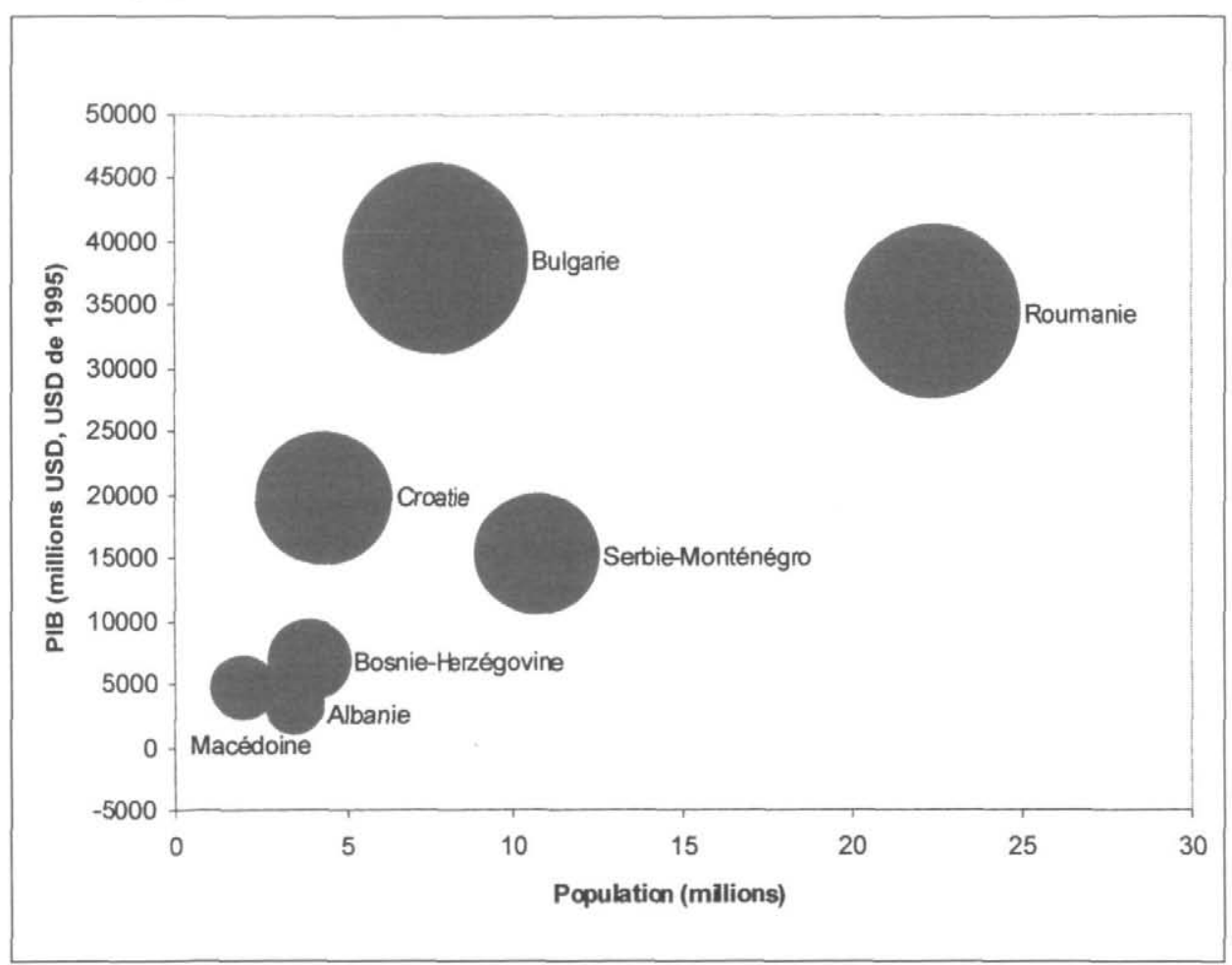

Source : calculé à partir des données de CHELEM

La figure 2 indique une forte inégalité dans les tailles et les niveaux de PIB des pays membres de la ZLE-Balkans signée en juin 2001. Etant plus restreints en valeur absolue, les marchés bosniaque, macédonien et albanais offrent peu de possibilité d'expansion et de création de commerce. A l'inverse, Bulgarie et Roumanie apparaissent comme des poids lourds de la ZLE et seront en mesure 
de tirer un profit plus important de cette dernière (débouchés plus importants pour les entreprises, rendements d'échelle plus marqués).

Quoiqu'il en soit, les effets de création et d'expansion de commerce risquent d'être limités en raison de la faible intensité du commerce mutuel au sein de la zone (figure3).

\section{Figure 3 : Commerce mutuel intra ZLE-Balkans en \% des exportations totales de chaque pays membre [1993 à 2000]}

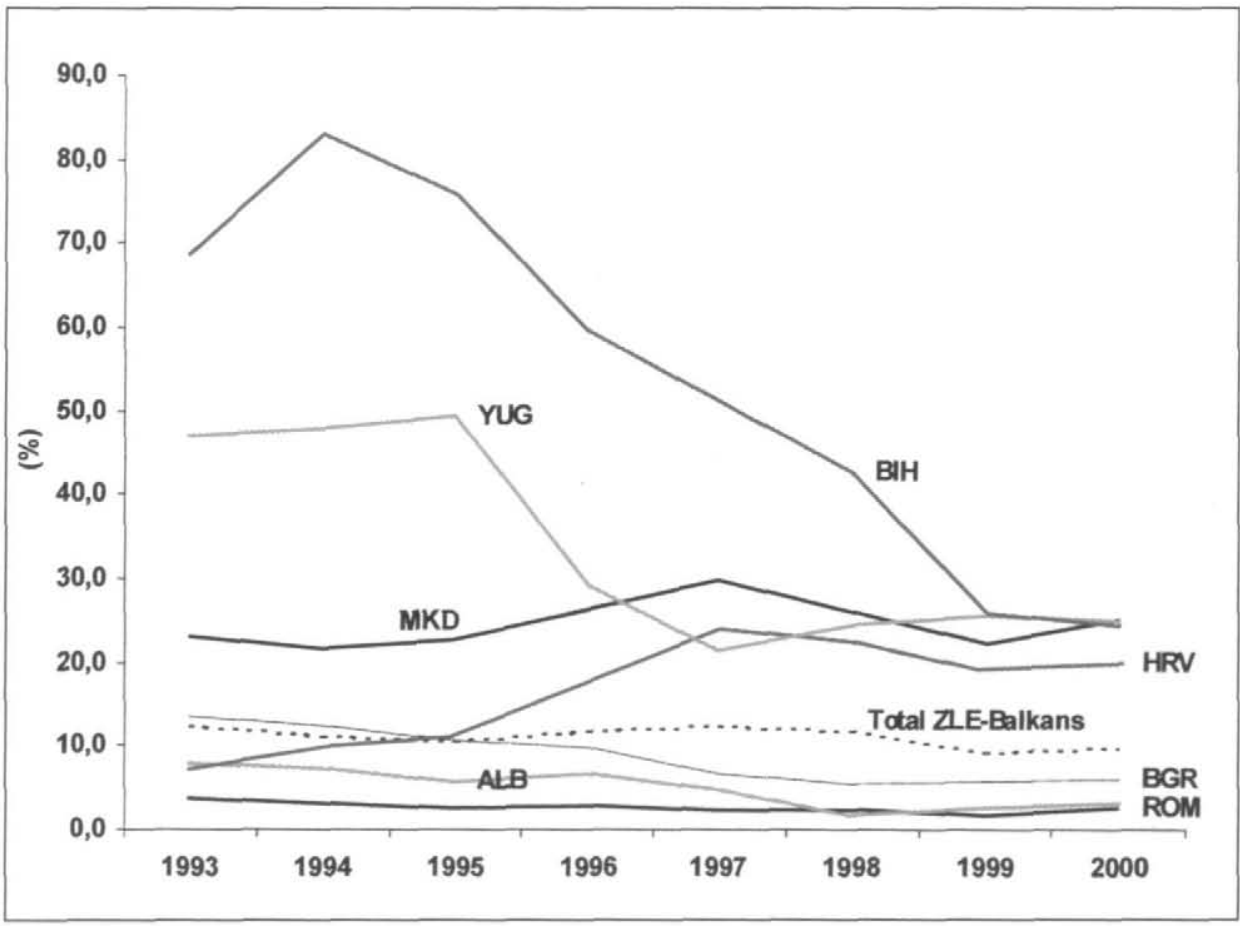

BIH : Bosnie-Herzégovine ; YUG : Serbie-Monténégro ; MKD : Macédoine ; HRV : Croatie ; BGR : Bulgarie ; ROM : Roumanie ; ALB : Albanie.

Source : calculé à partir des données de CHELEM

Le commerce mutuel s'est effondré durant la décennie 90 pour se situer à 9,7\% du commerce total des membres de la ZLE-Balkans en 2000. Cela veut dire que les effets commerciaux générés par la formation d'une zone de libreéchange ne pourront jouer qu'à la marge ${ }^{31}$. En 2000, le commerce intra-ZLEBalkans se répartissaient comme suit : 
Figure 4 : Origine des exportation intra-ZLE-Balkans en 2000

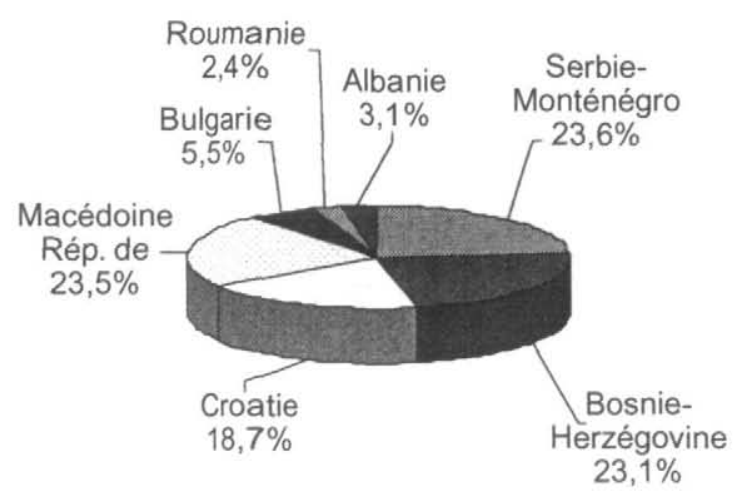

Les pays les plus importants et les plus développés (Croatie, Roumanie, Bulgarie) ne génèrent que $26,6 \%$ du commerce mutuel. Ajoutons à cela le fait que l'union est trop petite ( $0,3 \%$ du commerce mondial) pour influencer les prix mondiaux et pouvoir ainsi bénéficier d'effets prix ${ }^{32}$.

Ajoutons, en guise de conclusion sommaire, que l'UE occupe une place prépondérante dans le commerce extérieur des pays balkaniques. L'UE est le principal partenaire commercial de ces pays. L'UE absorbera deux poids lourds de la ZLE-Balkans. L'UE commence à signer des accords d'association avec les pays de l'ex-RFSY (hors Slovénie). C'est donc avec l'UE que le potentiel d'effets de création et de détournement d'échanges est le plus fort.

Tableau 3 : Matrice du commerce intra-balkanique (millions USD courants, 1993 à 2000)

\begin{tabular}{|l|l|c|c|r|c|c|c|c|c|}
\hline $\begin{array}{l}\text { Destination } \\
\text { Origine }\end{array}$ & Année & $\begin{array}{c}\text { Serbie- } \\
\text { Mont. }\end{array}$ & $\begin{array}{c}\text { Bosnie- } \\
\text { Herzégovine }\end{array}$ & Croatie & $\begin{array}{c}\text { Rép. de } \\
\text { Macédoine }\end{array}$ & Bulgarie & Roumanie & Albanie & $\begin{array}{c}\text { Total } \\
\text { Export. }\end{array}$ \\
\hline Serbie- & 1993 & 0 & 159,8 & 0,6 & 143,6 & 58,8 & 26,4 & 5,3 & 394,5 \\
\hline Monténégro & 1994 & 0 & 128,2 & 0,4 & 139,0 & 49,8 & 33,6 & 4,9 & 355,9 \\
\hline & 1995 & 0 & 137,7 & 0,4 & 184,0 & 61,6 & 35,1 & 5,6 & 424,4 \\
& 1996 & 0 & 126,9 & 0,4 & 181,0 & 55,9 & 40,2 & 6,6 & 411,0 \\
& 1997 & 0 & 149,2 & 24 & 196,5 & 36,4 & 49,6 & 1,5 & 457,2 \\
& 1998 & 0 & 201,1 & 15,7 & 247,5 & 35,8 & 51,1 & 8 & 559,2 \\
& 1999 & 0 & 50,3 & 16,3 & 142,8 & 29,2 & 38,2 & 10,5 & 287,3 \\
\hline & 2000 & 0 & 72,1 & 20,9 & 171,5 & 35,6 & 45,6 & 5,5 & 351,2 \\
\hline
\end{tabular}




\begin{tabular}{|c|c|c|c|c|c|c|c|c|c|}
\hline $\begin{array}{l}\text { Destination } \\
\text { Origine }\end{array}$ & Année & $\begin{array}{l}\text { Serbie- } \\
\text { Mont. }\end{array}$ & $\begin{array}{c}\text { Bosnie- } \\
\text { Herzégovine }\end{array}$ & Croatie & $\begin{array}{c}\text { Rép, de } \\
\text { Macédoine }\end{array}$ & Bulgarie & Roumanie & Albanie & $\begin{array}{c}\text { Total } \\
\text { Export. }\end{array}$ \\
\hline Bosnie- & 1993 & 133,5 & 0 & 13,5 & 0,9 & 0 & 9,4 & 0 & 157,3 \\
\hline \multirow[t]{7}{*}{ Herzégovine } & 1994 & 137,7 & 0 & 4,5 & 0,8 & 0 & 0 & 0 & 143,0 \\
\hline & 1995 & 142,6 & 0 & 8,4 & 0,8 & 0 & 0 & 0 & 151,8 \\
\hline & 1996 & 147,2 & 0 & 50 & 3,1 & 0,1 & 0,6 & 0 & 201,0 \\
\hline & 1997 & 181,5 & 0 & 108 & 4,4 & 0,3 & 4,9 & 0,1 & 299,2 \\
\hline & 1998 & 159,2 & 0 & 127 & 7,7 & 0,3 & 7,1 & 0,2 & 301,5 \\
\hline & 1999 & 70,6 & 0 & 104,3 & 5,3 & 0,4 & 4,1 & 0,2 & 184,9 \\
\hline & 2000 & 107,7 & 0 & 85,9 & 6,1 & 0,4 & 7,3 & 0,2 & 207,6 \\
\hline \multirow[t]{8}{*}{ Croatie } & 1993 & 7 & 182,9 & 0 & 53 & 4,3 & 3,3 & 6 & 256,5 \\
\hline & 1994 & 6,6 & 296,7 & 0 & 64,1 & 4,4 & 2,6 & 6,6 & 381,0 \\
\hline & 1995 & 6,8 & 395,6 & 0 & 60,8 & 5,8 & 3,4 & 7,1 & 479,5 \\
\hline & 1996 & 7,1 & 718,5 & 0 & 52,3 & 5 & 4,1 & 10,6 & 797,6 \\
\hline & 1997 & 23,8 & 894,9 & 0 & 62,1 & 9,7 & 14,8 & 4,5 & 1009,8 \\
\hline & 1998 & 11,0 & 934,4 & 0 & 64,3 & 8,2 & 4,8 & 5,4 & 1028,1 \\
\hline & 1999 & 11,4 & 686,6 & 0 & 57,4 & 7,8 & 4,5 & 7,5 & 775,2 \\
\hline & 2000 & 39,3 & 689,4 & 0 & 61,4 & 9,9 & 3,4 & 11,5 & 814,9 \\
\hline Rép de & 1993 & 136,3 & 2,8 & 38,6 & 0 & 96,0 & 15,6 & 16,0 & 305,3 \\
\hline \multirow[t]{7}{*}{ Macédoine } & 1994 & 140,6 & 4,2 & 27,3 & 0 & 96,1 & 3,7 & 16,8 & 288,7 \\
\hline & 1995 & 160,1 & 6,1 & 33,9 & 0 & 107,4 & 4,5 & 17,2 & 329,2 \\
\hline & 1996 & 218,0 & 17,8 & 32,8 & 0 & 32,6 & 3,7 & 23,2 & 328,1 \\
\hline & 1997 & 263,2 & 20,6 & 37,7 & 0 & 28,8 & 4,0 & 22,5 & 376,8 \\
\hline & 1998 & 236,2 & 22,2 & 53,7 & 0 & 31,1 & 4,9 & 4,4 & 352,5 \\
\hline & 1999 & 154,3 & 17,3 & 45,4 & 0 & 31,7 & 3,1 & 11,3 & 263,1 \\
\hline & 2000 & 199,9 & 18,4 & 48,3 & 0 & 36,7 & 2,6 & 27,4 & 333,3 \\
\hline \multirow[t]{8}{*}{ Bulgarie } & 1993 & 182,9 & 1,2 & 8,4 & 167,9 & 0 & 62,3 & 24,3 & 447,0 \\
\hline & 1994 & 191,1 & 1,5 & 10,2 & 201,6 & 0 & 58,0 & 26,2 & 488,6 \\
\hline & 1995 & 217,4 & 2,1 & 18,1 & 224,9 & 0 & 76,5 & 32,9 & 571,9 \\
\hline & 1996 & 214,6 & 3,0 & 12,8 & 121,2 & 0 & 72,0 & 38,1 & 461,7 \\
\hline & 1997 & 119,2 & 3,9 & 14,8 & 112,3 & 0 & 57,6 & 23,6 & 331,4 \\
\hline & 1998 & 88,0 & 3,9 & 10,1 & 86,0 & 0 & 49,6 & 20,6 & 258,2 \\
\hline & 1999 & 56,1 & 3,0 & 9,2 & 105,1 & 0 & 52,8 & 25,0 & 251,2 \\
\hline & 2000 & 75,5 & 3,3 & 9,1 & 123,6 & 0 & 77,7 & 23,9 & 313,1 \\
\hline \multirow[t]{8}{*}{ Roumanie } & 1993 & 72,4 & 2,6 & 3,6 & 15,8 & 76,1 & 0 & 9,6 & 180,1 \\
\hline & 1994 & 87,6 & 0,5 & 9,5 & 11,6 & 71,1 & 0 & 10,9 & 191,2 \\
\hline & 1995 & 97,6 & 0,7 & 11,2 & 11,2 & 73,8 & 0 & 13,4 & 207,9 \\
\hline & 1996 & 122,8 & 1,8 & 16,6 & 10,8 & 68,6 & 0 & 22,9 & 243,5 \\
\hline & 1997 & 110,0 & 4,8 & 24,6 & 12,2 & 50,4 & 0 & 2,8 & 204,8 \\
\hline & 1998 & 101,9 & 6,2 & 14,4 & 9,1 & 57,6 & 0 & 3,6 & 192,8 \\
\hline & 1999 & 56,3 & 4,6 & 12,3 & 11,0 & 80,1 & 0 & 5,5 & 169,8 \\
\hline & 2000 & 88,2 & 5,2 & 18,5 & 14,3 & 137,8 & 0 & 7,3 & 271,3 \\
\hline
\end{tabular}




\begin{tabular}{|l|l|r|r|r|r|r|r|r|r|}
\hline $\begin{array}{l}\text { Destination } \\
\text { Origine }\end{array}$ & Année & $\begin{array}{r}\text { Serbie- } \\
\text { Mont. }\end{array}$ & $\begin{array}{c}\text { Bosnie- } \\
\text { Herzégovine }\end{array}$ & Croatie & $\begin{array}{r}\text { Rép. de } \\
\text { Macédoine }\end{array}$ & Bulgarie & Roumanie & Albanie & $\begin{array}{r}\text { Total } \\
\text { Export. }\end{array}$ \\
\hline Albanie & 1993 & 2,2 & 0,1 & 1,6 & 5,7 & 0,1 & 0,1 & 0 & 9,8 \\
\hline & 1994 & 2,4 & 0,2 & 2,0 & 6,9 & 0,1 & 0,1 & 0 & 11,7 \\
& 1995 & 2,7 & 0,3 & 2,2 & 7,5 & 0,1 & 0,1 & 0 & 12,9 \\
& 1996 & 2,9 & 0,5 & 1,6 & 5,7 & 0,1 & 8,3 & 0 & 19,1 \\
& 1997 & 0,6 & 0,7 & 4,0 & 5,7 & 0,1 & 0,1 & 0 & 11,2 \\
& 1998 & 1,5 & 0,7 & 1,0 & 1,3 & 0,1 & 0,3 & 0 & 4,9 \\
& 1999 & 1,7 & 0,5 & 0,8 & 3,8 & 0,1 & 0,1 & 0 & 7,0 \\
& 2000 & 2,5 & 0,5 & 0,8 & 5,2 & 0,1 & 0,0 & 0 & 9,1 \\
\hline Total & 1993 & 534,3 & 349,4 & 66,3 & 386,9 & 235,3 & 117,1 & 61,2 & 1750,5 \\
importations & 1994 & 566,0 & 431,3 & 53,9 & 424,0 & 221,5 & 98,0 & 65,4 & 1860,1 \\
& 1995 & 627,2 & 542,5 & 74,2 & 489,2 & 248,7 & 119,6 & 76,2 & 2177,6 \\
& 1996 & 712,6 & 868,5 & 114,2 & 374,1 & 162,3 & 128,9 & 101,4 & 2462,0 \\
& 1997 & 698,3 & 1074,1 & 213,1 & 393,2 & 125,7 & 131,0 & 55,0 & 2690,4 \\
& 1998 & 597,8 & 1168,5 & 221,9 & 415,9 & 133,1 & 117,8 & 42,2 & 2697,2 \\
& 1999 & 350,4 & 762,3 & 188,3 & 325,4 & 149,3 & 102,8 & 60,0 & 1938,5 \\
\hline & 2000 & 513,1 & 788,9 & 183,5 & 382,1 & 220,5 & 136,6 & 75,8 & 2300,5 \\
\hline
\end{tabular}

Source : matrices calculées à partir de CHELEM

Tableau 4 : Exportations [FOB] totales de chacun des pays de la ZLE-Balkans (millions USD courants, 1993 à 2000)

\begin{tabular}{|l|l|r|r|r|r|r|r|r|r|}
\hline Exportateur & & \multicolumn{1}{|c|}{1993} & \multicolumn{1}{|c|}{1994} & \multicolumn{1}{c|}{1995} & \multicolumn{1}{c|}{1996} & \multicolumn{1}{c|}{1997} & \multicolumn{1}{c|}{1998} & \multicolumn{1}{c|}{1999} & \multicolumn{1}{c|}{2000} \\
\hline Serbie-Monténégro & YUG & 840,2 & 743,1 & 862,3 & 1413,6 & 2135,9 & 2273,6 & 1121,3 & 1394,3 \\
Bosnie-Herzégovine & BIH & 228,9 & 172,4 & 199,9 & 337,5 & 582,7 & 705,7 & 711,3 & 844,5 \\
\hline Croatie & HRV & 3522,6 & 3894,1 & 4252,6 & 4482,3 & 4223,6 & 4584,6 & 4051,4 & 4087,2 \\
Macédoine, Rép.de & MKD & 1318,1 & 1333,7 & 1438,5 & 1231,4 & 1270,9 & 1356 & 1182,6 & 1326,6 \\
Bulgarie & BGR & 3331,8 & 3938,6 & 5346,4 & 4763,1 & 4908,6 & 4758,8 & 4379,2 & 5266,9 \\
Roumanie & ROM & 4769,2 & 6125,5 & 8184,8 & 8400,9 & 8467,3 & 8699,6 & 9146,6 & 10603,9 \\
Albanie & ALB & 127,9 & 162,4 & 222,9 & 285,9 & 244,6 & 262,6 & 256 & 276,1 \\
\hline
\end{tabular}

